\title{
UCTx: A Multi-Agent system to assist a Transplant Coordination Unit
}

\author{
U. CORTÉS, J. VÁZQUEZ-SALCEDA \\ Departament de Llenguatges i Sistemes Informàtics. \\ Universitat Politècnica de Catalunya. \\ c/ Jordi Girona 1-3. E08034 Barcelona, Spain. \\ Phone: 34934017016 Fax: 34934017014 \\ $\{$ ia, jvazquez $\} @ l s i . u p c . e s$ \\ A. LÓPEZ-NAVIDAD, F. CABALLERO \\ Banc de Teixits. Hospital de la Santa Creu $i$ Sant Pau \\ c/ St. Antoni M. Claret, 16\%. E08025 Barcelona, Spain. \\ Phone 34932919335 Fax 34932919410
}

\{ alopeznavidad,fcaballero\} @hsp.santpau.es

\begin{abstract}
We present a system called UCTx designed to model and automate some of the tasks performed by a Transplant Coordination Unit (UCTx) inside a Hospital. The aim of this work is to show how a multi-agent approach allows us to describe and implement the model, and how UCTx is capable of dealing with another multi-agent system (Carrel, an Agent Mediated Institution for the Exchange of Human Tissues among Hospitals for Transplantation) in order to meet its own goals, acting as the representative of the hospital in the negotiation. As an example we introduce the use of this Agency in the case of Cornea Transplantation.
\end{abstract}

Keywords: Agent Mediated Institutions, Negotiation, Tissues Bank, Transplants.

\section{Introduction}

In the procurement of human organs, tissues and/or bones for transplantation the key role of a Transplant Coordination Unit (UCTx) attracted our attention because of its complexity. We follow the work of López-Navidad et al [16] to describe all those tasks. During a single procurement or transplant episode many professionals, involved in a variety of medical acts, administer medical care. In this process health care administration personnel, health care professionals and so- cial care professionals interact with health-related information that is complex, heterogeneous and, maybe, geographically distributed. We saw this UCTX as a good example of a multi-agent system that has to interact not only with another multi-agent systems as the Carrel institution [5] but also with other non-software agents, that is, actors of different nature such as: Donors, Surgeons, Human Transplant Coordinators and organizations as the Organització Catalana de Transplantaments (OCATT) and the Organización Nacional de Transplantes (ONT). ${ }^{1}$ [25]. Each agent 
has different needs in terms of information access, security, and quality of service, and is involved in different medical acts and healthcare procedures.

Carrel is an Agent Mediated Institution designed to allow the distribution of human organs, tissues and/or bones for transplantation. It is an Intelligent Resources Management Service. The central idea is to bring all the information from the different Tissue Banks into Carrel, so Agents representing different Hospitals can access the Institution and negotiate for the pieces a given Hospital needs for a given transplantation. Those Agents have to accept Carrel's norms of negotiation. This fact determines the co-operative nature of the Institution.

\subsection{Related work}

Medical Domain has been always a fertile area for Artificial Intelligence applications. In the last years sophisticated knowledge-based systems have been introduced and there exists a lot of literature on applying different techniques, tools and available knowledge to enrich the reasoning process of those systems, see for example [11]. But this is not the case for transplantation, so we have to relay in the re-use of solutions/approaches to try to solve our problem.

For the Knowledge Representation and Ontology construction for the medical domain we follow the work developed in GRAIL [29]. A comprehensive set of Internet resources for medical terminology is compiled in [18].

The work of Decker [7] on the design of coordination mechanisms for groups of agents applied to Hospital scheduling is clear antecedent for our work. For planning in the medical domain we follow the work of Miksch [19]. The ideas about the implementation of medical protocols follow the work of Pattison-Gordon et al [26]. A related approach for monitoring medical protocols is described in [2]. We do not have news from other multi-agent systems applied to Transplants.

For a good survey on Autonomus Agents in Health Care see [30] and, the work of Lanzola et al related to cooperative agents in Medical applications [13].

\subsection{Organization of this paper}

In section $\S 2$ we explain in detail the architecture of UCTx and we briefly address some security issues. In particular, in $\$ 2.2$, we explain the exchanges of information among the different agents.

In section $\S 3$ we give a practical example of the possible application of UCTx studying the case of Cornea Transplantation, including an explanation and examples of the Selection Function introduced in $\S 3.1$. Finally, in $\S 4$, we present some conclusions.

\section{UCTx: The Transplant Coordination Agency}

Our implementation of an $U C T x$ has to reflect the infrastructure and staff of a real Transplant Coordination Unit which allows the successful conclusion of an organ or tissue ${ }^{2}$ procurement and extraction process for transplantation [16]. But it also includes new procedures designed to support the distributed computational system. In addition, it deals with the management of the requests for pieces made by the surgeons in order to transplant them into recipients and, it has to follow the sanitary and economic policies that the Hospital dictates.

The guidelines for this work are the existing protocols in the Hospital de la Santa Creu i Sant Pau, in Barcelona. Guideline could be defined as a method that identifies actions to be performed and conditions that govern when it is appropriate to perform them [26]. Protocols are important for improving quality assurance, for standardization in clinical practice, for guiding data collection, for better management, for improving decision support. All these reasons support our choice to create $U C T x$ as a multi-agent system where each agent adopts a role and implements one or more protocols or its tasks help in the implementation of a protocol. And we are expecting that those protocols are shared by any other $U C T x$ implementation or by any Transplant Coordination Unit.

The UCT $x$ implementation has been developed in $\mathrm{JADE}^{3}[3]$, a framework to create agent platforms that implements all the agent management and communication protocols, leaving to programmers the definition of the agents' internal logic 
and their behaviors. Each hospital owns a JADE platform (a UCTx platform) that communicates with Carrel. We expect to have UCTx and Carrel communication fully operational in June 2001. Other actors as ONT and OCATT will be connected later.

Transplants information is considered high-risk as it includes sensitive information about people (donors and recipients). So the UCTx and Carrel systems have to observe the local, national and European Union legislation on transplants (see the reports of the ONT in [6] and the recommendations of the Transplant Experts Committee in [17]). It also follows the European directives and the Spanish law on personal data protection [15] [9] [28]. In particular, both have to assure confidentiality and integrity of patient and donor's data and privacy. This is a long-standing issue in health care that acquires new facets with use of Electronic Medical Records (EMR). One of the benefits of using electronic records is that it assures access for authorized and authenticated users and helps keeping track as demanded by law. Donors and patients have to trust that the information about them may not be used for any purpose beyond that for which it was collected by UCTx. To compare the situation in US see for example the report elaborated by the Institute of Medicine [8].

UCTx is an Agency designed to be included among the public services that AgentCities initiative will offer [1]. AgentCities is an EU funded research project.

\subsection{Description of the Agency}

A UCTx is modeled as an Agency (see figure 1) that has several agents, each one competent in a specific task and with its own role and goals to achieve [27]. The Agency is composed of a Coordinator Agent, Surgeon, Analyzer, Finder, Planner, Consultation and Arrival agents.

We can identify the following fundamental services that $U C T x$ should provide to those agents:

1. Information confidentiality

2. Information integrity

3. Dynamic accessibility
The first two items are related to data security. As each (software or non-software) agent has a certain role in the system, we are choosing a rolebased access mechanism for data confidentiality control [14] (that is, different roles have different views of the data). The data integrity is delegated to the data base controller. The last item is related with the challenge to design and develop clinical systems which are intuitive to use and adequately expressive to satisfy a number of broad requirements: clinical generality, medical expressiveness, operational flexibility, soundness and safety, verifiability and support for reusability. There exist some efforts in this line as GRAIL [29] or PRoforma [10] that we will follow.

The Surgeon Agent, specific for each kind of piece, is responsible of communicating with the surgeons through the Surgeon Interface and it collects and formalizes the requests for pieces for transplant. Each request has to include the relevant information about the patient, the required piece, optional medical and economic restrictions and the Selection Function. The Surgeon Agent is able to specialize the Selection Function for a given patient using the relevant information coming from the surgeon and the patient's data (see §3.1). This function provides a way to evaluate each piece for a given offer in the Institution ${ }^{4}$ for Human Tissue Exchange. The Institution, in this case Carrel [5], assigns the pieces to the different competitor finder agents, one for each hospital and piece, maximizing the satisfaction degree of each request.

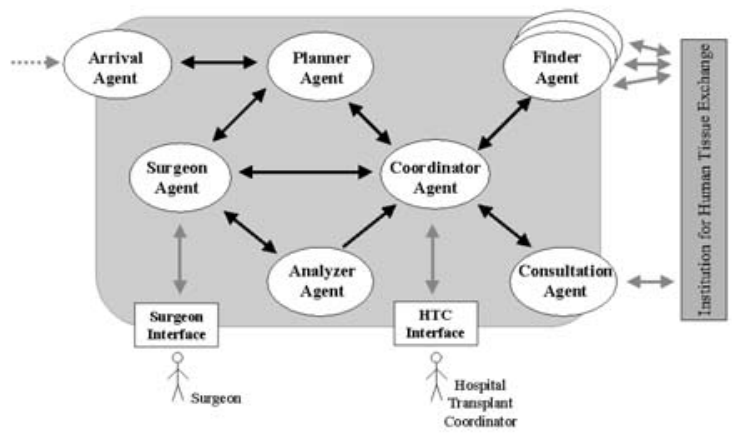

Fig. 1. The Transplant Coordination Unit's Agency 
The Surgeon Agent sends the request to the $A n$ alyzer Agent (see message $\mathrm{H} 1$ in table 1). This agent, specific for each kind of piece, will check if the information was properly introduced, that is, if all the characteristics needed were entered, and if the values are consistent following a given protocol. If there is some data missing, it informs the Surgeon Agent who will ask the surgeon to enter or to modify the data in order to validate it. When the Analyzer has all the information required, it sends the request to the Coordinator Agent (see message $\mathrm{H} 3$ in table 3 ).

The Coordinator Agent is responsible for the distribution and coordination of the different tasks that make up the whole process. The Hospital Transplant Coordinator in person can communicate with this Agent through the Coordinator Interface, and control the behavior of the agent. The Coordinator Agent has the following tasks:

1. to coordinate all the agents involved in each transaction.

2. to check all the surgeons' requests for tissues to ensure they meet the hospital protocols.

3 . to create, for each checked request, a new Finder Agent (see message $\mathrm{H} 4$ in table 4), that will be the one going to the Institution to look for the desired piece. The Coordinator Agent will give to the Finder Agent the request inside an electronic sealed envelope (see 2.2).

4. to keep records of all the piece requests made by the hospital in representation of its surgeons.

5. to give feed-back to the Institution (see [5]) when the piece arrives, after transplantation and three weeks after the operation, or in the case of any fatality.

6. to give feed-back of all the information about tissue requests (surgeon who made the request, request status and so on) the Hospital Transplant Coordinator wants to know in real-time or also by a query in the Coordinator Interface. All the feedback is displayed through the mentioned Coordinator Interface, which also allows the Hospital Transplant $\mathrm{Co}$ ordinator to set the amount of real-time information he wants to be displayed.

7. certify that any access to the hospital's medical data is performed by an authorized agent.
8. to create, for each query requesting information about tissues, a new Consultation Agent (see message $\mathrm{H} 13$ in table 7 ), that will be the one going to the Institution to ask for the desired information.

The Finder Agent is provided with a sealed envelope (see 2.2 ) with all the information required (ie. hospital information, patient's data, selection function, etc). When a Finder Agent returns from Carrel, it communicates the result of the negotiation to the Coordinator Agent (see message H7 in table 5). If a piece is found, the Coordinator Agent, as part of its agent coordination task, passes the delivery plan proposed by the Institution and the relevant information about the request to the Planning Agent (see message H17 in table 11), which will make up a logistic plan for the reception and transplantation. This information has to arrive to the surgeon that will perform the transplant, too. If no piece was found, the Coordinator asks the Surgeon Agent to inform the surgeon of such failure. The surgeon can then revise and resubmit the request, or perhaps this can provoke an impasse situation that can only be resolved by the Hospital Transplant Coordinator in person. The Surgeon and the Coordinator agents can stop the process of a request at any moment, if needed.

The Planner Agent is responsible for creating the transplant plan, that is, finding a surgery room to match the arrival time of the piece and the surgeon's available schedule. The Planner Agent can send several proposals to the surgeon through the Surgeon Agent (see message H15 in table 9). When the surgeon agrees with one of them, the Planner will carry out the transplant plan and will also send a message to the Coordinator Agent. Otherwise, the Surgeon Agent can re-use a given proposal or to create its own proposal and then send it to the Planner. If the proposal cannot be carried out, the Planner can ask for help to the Hospital Transplant Coordinator in person and/or notify the problem to the surgeon. The special characteristics of planning in the Medical Domain are discussed by Miksch [19]. We are following Decker's approach for task planning [7].

The Arrival Agent is responsible for updating the Planner Agent about events that can change the delivery plan (see messages H23 and H24 in 
Table 1. Messages Surgeon Agent $\rightarrow$ Analyzer Agent

\begin{tabular}{|c|c|c|}
\hline Message $\sharp$ & Predicate & Parameters \\
\hline H1 & piece_request & $\begin{array}{l}\text { id_request, id_piece, piece_params, } \\
\text { info_recipient, preferences, urgency_level }\end{array}$ \\
\hline
\end{tabular}

Table 2. Messages Analyzer Agent $\rightarrow$ Surgeon Agent

\begin{tabular}{|l|l|}
\hline Message $\sharp$ Predicate & Parameters \\
\hline H2 $\mid$ analysis_result & $\mid$ id_request, ok | (error, reason) \\
\hline
\end{tabular}

Table 3. Messages Analyzer Agent $\rightarrow$ Coordinator Agent

\begin{tabular}{|c|c|c|}
\hline Message $\sharp$ & Predicate & Parameters \\
\hline $\mathrm{H} 3$ & valid_piece_request & $\begin{array}{l}\text { id_request, id_piece, piece_params, } \\
\text { info_recipient, preferences, urgency_level }\end{array}$ \\
\hline
\end{tabular}

Table 4. Messages Coordinator Agent $\rightarrow$ Finder Agent

\begin{tabular}{|c|c|c|}
\hline Message $\sharp$ & Predicate & Parameters \\
\hline $\mathrm{H} 4$ & init_request & $\begin{array}{l}\text { id_request, id_piece, piece_params, } \\
\text { info_recipient, preferences, urgency_level }\end{array}$ \\
\hline H5 & stop_request & id_request, reason \\
\hline H6 & piece_problem_answer & id_request, neн_preferences | accept | refuse \\
\hline
\end{tabular}

Table 5. Messages Finder Agent $\rightarrow$ Coordinator Agent

\begin{tabular}{|c|c|c|}
\hline Message $\sharp$ & Predicate & Parameters \\
\hline $\mathrm{H} 7$ & piece_assignation & id_request, (piece_info, delivery_plan) | none \\
\hline H8 & piece_problem_question & id_request, info_problem \\
\hline
\end{tabular}

table 13 ), events that can occur while the transportation of the tissue is made from the Tissue Bank to the Hospital. Then the Planner will be able to modify the delivery or the transplant plans dynamically. That is of special interest when there are problems with the delivery (for example, when the hospital receives the wrong piece or the received piece is defectous).

Finally the Consultation Agent, which is the interface with the Institution's database, processes the different types of queries sent by the Surgeon Agent, the Planner Agent or the Coordinator Agent. This is done, as Finder Agents do, by going to the Carrel institution. As the Carrel consul- 
Table 6. Messages Surgeon Agent $\rightarrow$ Coordinator Agent

\begin{tabular}{|c|c|c|}
\hline Message $\sharp$ & Predicate & Parameters \\
\hline H9 & piece_problem_answer & id_request, neн_preferences | accept | refuse \\
\hline H10 & query_request & id_query, info_request \\
\hline H11 & transplant_info & id_request, transplant_data \\
\hline $\mathrm{H} 12$ & stop_request & id_request, reason \\
\hline
\end{tabular}

Table 7. Messages Coordinator Agent $\rightarrow$ Consultation Agent

\begin{tabular}{|l|l|}
\hline Message $\sharp \mid$ Predicate & Parameters \\
\hline H13 | query_request & $\mid$ id_query, info_request \\
\hline
\end{tabular}

tation procedures also follow the role-based access model, different levels of privilege are defined by the Carrel institution to restrict the access to its database, so, for instance, queries created in the Coordinator Agent have a higher privilege level than the ones created inside the Surgeon Agent or the Planner Agent, and having a higher privilege level means having access to a wider amount of information in the Institution's database. The Consultation Agent has to cope with this privileged levels and the possible rejection of all or part of the information requested.

\subsection{The Envelope}

The information required by the Finder Agent to look for a piece in the Carrel is packed in an electronic Sealed Envelope. This envelope is created by the Coordinator Agent after it has received a valid piece request from the Surgeon Agent. The envelope contains the following information:

- Urgency level, that works as electronic postage stamp and sets the urgency level of the request (in Spain: normal, urgency-1 or urgency-0)

- Hospital identification, together with the coordinator's electronic signature. This electronic signature is an electronic certificate issued by the Certification Authority associ- ated to the Carrel institution [5], and allows that institution to authenticate each request sender to make sure that only Finder Agents with requests from authorized senders can enter and negotiate inside Carrel.

- Piece information (type, parameters, etc.) and recipient data (age, sex, laboratory analysis, etc.).

- The selection function, as explained in section $\$ 3.1$.

The data needed by the Coordinator Agent in order to create this envelope comes from different sources that may be geographically distributed. The hospital identification and the electronic signature are issued by Carrel and only known by the Coordinator Agent. All the medical data about the piece and recipient are provided by the Surgeon Agent and the Analyzer Agent. An important constraint to be considered is the expected date for the transplant.

The format of an sealed envelope containing all the required information is the following: 
Table 8. Messages Consultation Agent $\rightarrow$ Coordinator Agent

\begin{tabular}{|l|l|}
\hline Message $\sharp \mid$ Predicate & Parameters \\
\hline H14 | query_result & | id_query, info_result \\
\hline
\end{tabular}

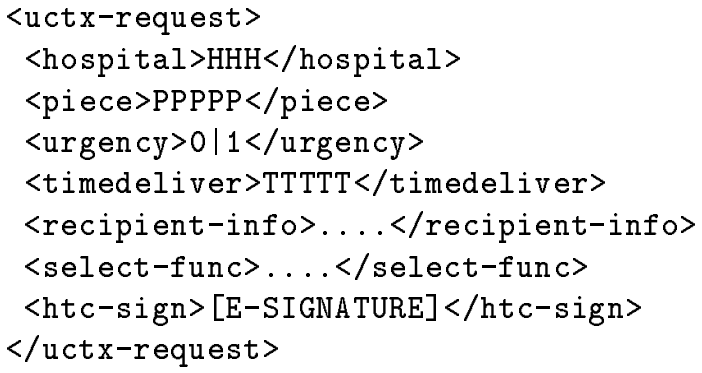

An UCTx Request Envelope

HHH stands for hospital identification code (which is provided by Carrel), PPPPP is the desired piece identification code and urgency $=0$ means delivery in less than 24 hours. TTTTT is the maximum date the recipient can wait to receive the piece (if applicable). The <recipient-info> tag has associated the recipient's relevant information, and the <selec-func > tag contains the Selection Function (see $§ 3.1$ ), which is private information. The envelope also contains the Hospital Transplant Coordinator's electronic signature.

The information required to create the selection function comes in part from the Surgeon Agent, reflecting the surgeon's preferences for the piece to get, and in part from the Coordinator Agent, reflecting the coordinator or hospital's preferences (such as costs, preferred Tissue Banks, etc.).

Once the envelope is created, it is delivered to the Finder Agent, which will send it to the Institution to look for the piece.

The envelope is an important piece of information that helps to protect the recipient's medical data and to improve and speed the assignment process. The envelope contains almost all the necessary information for the Finder Agent to perform its tasks.

\section{An Example: The Cornea Transplanta- tion}

We will use the Cornea Transplantation process to illustrate our ideas. Unlike most tissues in the body, the cornea contains no blood vessels. The cornea must remain transparent to refract light properly, and the presence of even the tiniest blood vessels can interfere with this process. To see properly, all layers of the cornea must be free of any cloudy or opaque areas. The cornea is as smooth and clear as glass and it helps the eye in two ways:

- It helps to shield the rest of the eye from germs, dust, and other harmful matter. The cornea shares this protective task with the eyelids, the eye socket, tears, and the sclera (see figure 2).

- The cornea acts as the eye's outermost lens. It functions like a window that controls and focuses the entry of light into the eye. The cornea contributes between $65-75 \%$ of the eye's total focusing power.

A corneal transplant involves replacing a diseased or scarred cornea with a new one. In corneal transplant surgery, the surgeon removes the central portion of the cloudy cornea and replaces it with a clear cornea (see figure 2), usually donated through a Tissue Bank (TB). A trephine is used to remove the damaged cornea. The surgeon places the new cornea in the opening and sews around it to connect it.

The need to automate part of the transplant coordination procedures can be seen if we take a look to the statistics show: in the United States over 40,000 cornea transplants are performed each year (in 1996 there were 46300 , that is, $178 \mathrm{pmp}^{5}$ ) [22], [4], [20]; in Catalonia the number of transplants is increasing: in 1999, 845 cornea transplants were performed, that is, $141 \mathrm{pmp}$, and in 2000 there were 929 transplants, that is, 152 pmp [25]. So the number of transplants is growing so much that 
Table 9. Messages Planner Agent $\rightarrow$ Surgeon Agent

\begin{tabular}{l|l|l|}
\hline Message $\sharp \mid$ Predicate & Parameters \\
\hline H15 | ask_surgeon_availability & id_request, \{time_ini, time_end $\}$ *, id_piece \\
\hline
\end{tabular}

Table 10. Messages Surgeon Agent $\rightarrow$ Planner Agent

\begin{tabular}{|l|l|}
\hline Message $\sharp$ Predicate & Parameters \\
\hline H16 $\mid$ tell_surgeon_availability $\mid$ id_request, time_ini, time_end*, id_piece
\end{tabular}

Table 11. Messages Coordinator Agent $\rightarrow$ Planner Agent

\begin{tabular}{|c|c|c|}
\hline Message $\sharp$ & Predicate & Parameters \\
\hline $\mathrm{H} 17$ & planning_request & id_request, delivery_plan, info_request \\
\hline $\mathrm{H} 18$ & query_request & id_request, info_request \\
\hline H19 & query_result & id_request, info_result \\
\hline
\end{tabular}

Table 12. Messages Planner Agent $\rightarrow$ Coordinator Agent

\begin{tabular}{|c|c|c|}
\hline | Message $\sharp$ & Predicate & Parameters \\
\hline $\mathrm{H} 20$ & planning_answer & id_request, deliveryplan, transplant_plant \\
\hline $\mathrm{H} 21$ & query_request & id_request, info_request \\
\hline $\mathrm{H} 22$ & query_result & id_request, info_result \\
\hline
\end{tabular}

Table 13. Messages Arrival Agent $\rightarrow$ Planner Agent

\begin{tabular}{|c|c|c|}
\hline Message $\sharp$ & Predicate & Parameters \\
\hline $\mathrm{H} 23$ & piece_reception & id_request, id_piece, state \\
\hline $\mathrm{H} 24$ & transportproblem & id_request, info_problem \\
\hline
\end{tabular}

Human Transplant Coordinators are beginning to be overwhelmed by the requests.

The chances of success of this operation have also risen dramatically because of technological advances in the procurement, examination, preservation and implantation procedures and the improvement of the post-implant treatments. For instance, a study supported by the National Eye Institute (NEI) suggests that matching the blood type, but not tissue type, of the recipient with that of the cornea donor may improve the success rate of corneal transplants in people at high risk for graft failure. In US, approximately $20 \%$ of corneal 


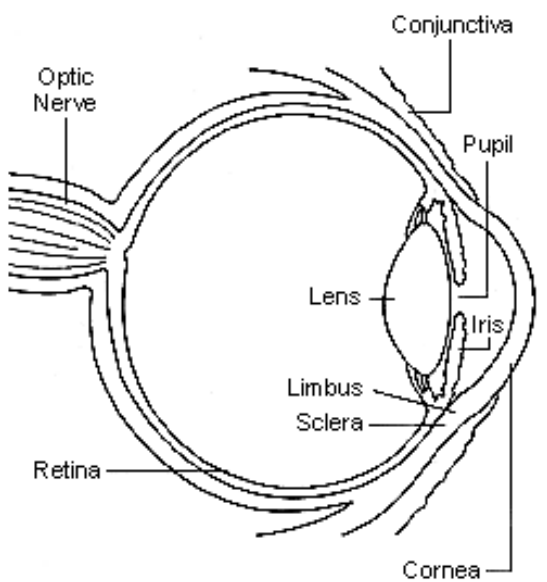

Fig. 2. The Eye

transplant patients-between $6000-8000$ a yearreject their corneal grafts. The NEI-supported study, called the Collaborative Corneal Transplantation Study [12], also concluded that intensive steroid treatment after transplant surgery improves the chances for a successful transplant.

So the aim of the UCTx system is not only to automate an important part of the procedures but also allow faster and more exhaustive ways to find the proper cornea ${ }^{6}$ for a given recipient, and also store data to monitor the whole process and to make further analysis of the results to detect any critical point in the procedures and then make the suitable improvements.

\subsection{The Selection Function}

Here we introduce the description of a Selection Function, one of the items that comprise the envelope's content the Finder Agent carries to the Institution with a request for a cornea. The Selection Function is a private piece of knowledge given by surgeons to guide the search for suitable corneas made by the Finder Agent.

The Selection Function is composed of a set of rules, each one a constraint the selected cornea has to satisfy. Some of these rules belong to the policy of the whole transplant unit of the hospital, and the rest of the rules are introduced by the surgeon, who can set the constraints needed for a given recipient.
A rule of the Selection Function can include:

- predicates about the piece: predicates that describe the constraints the selected cornea has to satisfy, such as the age of the donor or the density of Epithelial cells in the cornea.

- predicates about the Tissues Bank: predicates that can set constraints about the Tissue Bank preferred by the surgeon or the hospital. - predicates about the cost of the cornea: a predicate that can set a maximum cost for the selected cornea. This cost is related only to the cost of the cornea extraction and preservation process, and it is paid through a clearing house by the hospital who receives the cornea. An example of such predicate is $(<$ Cost 600euros).

As an example let us describe an imaginary recipient $r$ with the predicate rule $P_{r}$ as:

$$
\begin{aligned}
& P_{r}=\left\{\left(=\text { Age }_{r} \text { Young }\right) \wedge\left(=\text { Blood_Type }_{r} \text { A }\right)\right. \\
& \left.\wedge\left(=\text { Sex } x_{r} \text { Male }\right) \wedge \ldots\right\}
\end{aligned}
$$

and he needs a cornea for transplantation. The UCTx will prepare an envelope with the petition that will include the encrypted recipient information shown in 3.1 and the Selection Function shown in 3.2. This will be carried by the Finder Agent to the Institution [5].

$$
\begin{aligned}
& \left(=\text { Age }_{d} \text { Young }\right) \wedge(=\text { TB HSCSP }) \\
& \wedge\left(>E C_{d} 2000 / \mathrm{mm}^{2}\right)
\end{aligned}
$$

where $A g e_{d}$ stands for the donor's age, TB stands for Tissue Bank (in the example, HSCSP is the bank of the Hospital de la Santa Creu i Sant Pau) and $E C_{d}$ stands for the Endothelial Cells density in the donor's cornea. The definition of concepts like Young is settled by the Transplant Coordinator or by the Surgeons using an Ontology that is unique for each Hospital. Of course, each institution could specialize and customize its Selection Functions to fit with their policies. For example, in those countries where donors are in limited supply, to ask for ( $>E C_{d} 2000 / \mathrm{mm}^{2}$ ) and $\left(=\right.$ Age $_{d}$ Young) may exclude all available corneas in a TB so the UCTx should lower these constrains to some other more acceptable.

If we modify the recipient characteristics in 3.1 by doing $\left(=A g e_{r}\right.$ Old $)$ then we can have the 
following Selection function:

$$
(=T B H S C S P) \wedge\left(>E C_{d} 2000 / \mathrm{mm}^{2}\right)
$$

which in turn is more flexible than 3.2.

As each kind of transplant procedure (Cornea Transplant, Lamelar Transplant, Keratoconus Transplant... ) has different needs, there will be different rules for each one, and this means different selection functions. If we add to 3.1 the following information (= Transplant $K$ ), where $K$ stand for Keratoconus, then 3.2 will change to:

$$
\begin{aligned}
& \left(=A g e_{d} \text { Young }\right) \wedge(=T B H S C S P) \\
& \wedge\left(>E C_{d} 2800 / \mathrm{mm}^{2}\right)
\end{aligned}
$$

or even to

$$
\begin{aligned}
& \left(=\text { Age }_{d} \text { Young }\right) \wedge(=T B H S C S P) \\
& \wedge\left(>E C_{d} 2800 / \mathrm{mm}^{2}\right) \\
& \wedge\left(=\text { Erosion_E } p_{d} \text { False }\right)
\end{aligned}
$$

where Erosion_E $p_{d}$ expresses whether there is erosion in the donor's Epithelial layer of the cornea. It is possible to specialize $3.2,3,4$ and 5 by adding the following predicates

$$
\left(=H L A_{d} D R\right) \wedge\left(=B_{l o o d} T_{-T y p e} A B 0\right)
$$

The $H L A$ predicate will measure the histocompatibility between the Donor and the Recipient, although this is only important when a potential recipient had suffered from previous graft rejections.

Surgeons or the Hospital Transplant Coordinator can introduce other constraint rules about the cornea, such as the time it has been in preservation at the Tissues Bank:

$$
\left(=\text { Age }_{r} \text { Young }\right) \wedge(<\text { Hours_In_TB 72) }
$$

as some surgeons think that corneas with more than 3 days ( 72 hours) inside the TB are not good choices for a young recipient.

The surgeons can easily create their own rules to build their own selection functions by means of a rule editor in the Surgeon Interface. With this editor a surgeon can compose a rule, and then associate a weight to each rule. This interface keeps all rules to be re-used in future requests. This rule base will constitute the memory of each service and should be exploited to learn better strategies to create new rules. The weights associated to rules allow the Finder Agent to know which of the rules are more important than others while it is searching for a cornea and, as it was introduced in section $\S 2.1$, the weights allow to qualify each piece and create a partial order among them.

\subsection{Knowledge Representation}

The Medical Knowledge domain is very large and vast amounts of information are available in very different formats, but this knowledge is oftenly uncertain and vague. Another characteristic of this knowledge domain is that often it is incomplete and the sources of information are not always available in electronic format to allow evaluation processes.

Knowledge-based systems that support health care require large controlled terminologies to manage names and meanings of data elements. These terminologies are not static. To share data and applications in health care, we need standards and methods not only for terminologies and concept representation, but also for representing change [23]. This representation should be used in very different tasks as: a) search for a term or piece, b) retrieve information about a piece, a resource, etc., c) translation to other coding schemes (ie. between to hospitals), e) uniform edition of the knowledge base.

As the basis of our Knowledge representation we are choosing to re-use previous efforts as GRAIL [29] or PROforma [10] and to use, at this stage, a very simple language to build rules as those of $\S 3.1$. In the near future we expect to improve the expresivity of this language.

\section{Conclusions}

The social pressure and the need to improve the quality of health care has lead to a strong demand for clinical protocols and, lately, computer systems supporting both their creation and the execution. Organ and Tissue transplants in general, and corneal transplants in particular oftenly are the best technique for the treatment of some major health problems that can affect the quality of life of an important part of the population. So improving the success of such techniques is very important. 
In this work we propose the $U C T x$ agency, a Multi-Agent System that models the interaction of the different actors of a Transplant Coordination Unit inside a Hospital. The UCTx system interacts with an Agent Mediated Institution for Human Tissue Exchange (see Carrel in [5]), and this collaboration ensures that the process meets the protocols and the rules established by national transplant organizations and hospitals. $U C T x$ speeds up the process by its automation, which can reduce the time since the extraction of the tissue to its implant in the recipient, increasing the quality of the piece implanted. That is of special relevance in the cornea's transplantation, as corneas are perishable. In other cases, the use of the systems will help in finding the best stored piece for a given patient.

The UCTx platform can be very helpful in a Transplant Coordination Unit as it can aid in some of the daily management issues such as coordination of surgeons or planning of operations and even automate some tedious tasks such as looking for an available surgery room or more specialized as looking for a proper tissue. It will help to maintain the medical data integrity and confidentiality by supervising every access to data and keeping track of any data demand, see for example [24]. This is coherent with the new Spanish law on personnel data protection [15] [28] and meets also the European Directive 97/66/CE on Data Protection [9].

UCTx can assist the surgeons while they build their requests for pieces (see $\$ 3$ ), manage the requests and inform the Hospital Transplant Coordinator of any important event that occurs.

On the other hand, as the system asks for a complete clinical evaluation of each piece, it can decrease the cost of transplants by reducing the number of unsuitable transplants and furthermore offering a higher security level in reducing the chances of possible infections.

One additional advantage of using such a system is that the information that UCTx gathers about organs, tissues, bones and recipients is organized using an electronic format that can be analyzed later to obtain through data mining or machine learning procedures. Discovery of new knowledge by mining medical databases is crucial in order to make an effective use of stored data to generate new useful knowledge about the trans- plant process, knowledge which can lead to its improvement, from the enhancement of the tissue selection functions to the extraction and implantation procedures.

\section{Notes}

1. The Organización Nacional de Transplantes is a technical organization within the Spanish Department of Health and Consumer Affairs, without attributes of direct management and whose fundamental mission is the promotion, facilitation and coordination of all types of organs, tissues and bone marrow.

2. From now on we will use the word pieces to designate organs or tissues or bones.

3. JADE stands for Java Agent DEvelopment framework, a Java-based environment that follows the FIPA specifications for agent management, ACL message structure, communicative acts and interaction protocols.

4. Here we follow North's definition of an Institution as a collection of artificial constrains that shape human interaction [21].

5. pmp stands for per million of population.

6. We should note here that cornea transplants are only an example of use of the UCTx system, as it is obvious that a similar analysis can be done to use $U C T x$ with other kind of tissues such as skin or bones.

\section{References}

1. AgentCities. http://www.agentcities.org, 2000.

2. T. Alsinet, R. Béjar, C. Fernàndez, and F. Manyà. A multi agent system architecture for monitoring medical protocols. In C. Sierra and M. Gini J. Rosenschein, editors, Proceedings of the Fourth International Conference on Autonomous Agents, pages 499505. ACM-AAAI, ACM, 2000.

3. F. Bellfamine, A. Poggi, and G. Rimassa. JADE : A FIPA compliant agent frame-work. In Proc. PAAM'1999, pages 97-108, 1999.

4. F.S. Brightbill, editor. Corneal surgery: Theory, techniques and tissue. Mosby Inc., 1999.

5. U. Cortés, A. López-Navidad, J. Vázquez-Salceda, A. Vázquez, D. Busquets, M. Nicolás, S. Lopes, F. Vázquez, and F. Caballero. Carrel: An agent mediated institution for the exchange of human tissues among hospitals for transplantation. In $z^{e r}$ Congrés Català d'Intel.ligencia Artificial, pages 15-22. ACIA, 2000.

6. Organización Nacional de Transplantes, editor. Informes y Documentos de Consenso promovidos por la Organización Nacional de Transplantes y la Comisión de Transplantes del Consejo Interterritorial del Sistema Nacional de Salud. Editorial Complutense S. A., $1^{\text {st }}$ edition, 2000.

7. K. S. Decker. Task Environment Centered Simulation, chapter 6, pages 105-128. In Prietula et al. [27], $1^{\text {st }}$ edition, 1998. 
8. M. S. Donaldson and K. N. Lohr, editors. Health Data in the Information Age: Use, Disclosure, and Privacy. National Academy Press., Washington, D.C., $1^{\text {st }}$ edition, 1994. Institute of Medicine.

9. Directive 95/46/CE of the European Parliament and of the Council of 24 october 1995 on the protection of individuals with regard to the processing of personal data and of the free movement of such data, October 1995.

10. J. Fox, N. Johns, A. Rahmanzadeh, and R. Thompson. Proforma: A method and a language for specifying clinical guidelines and protocols. In J. Brender, J. P. Christensen, J-R. Scherrer, and P. McNair, editors, Medical Informatics Europe'96, pages 516-520. IOS Press, 1996.

11. L. Godo, R.López de Mántaras, J. Puyol-Gruart, and C. Sierra. Renoir, pneumon-ia and terap-ia: three medical applications based on fuzzy logic. Artificial Intelligence in Medicine, 21(1-3):153-162, 2001.

12. Collaborative Corneal Transplantation Studies Group. Collaborative corneal transplantation studies (CCTS). Technical report, National Eye Institute, 1999.

13. G. Lanzola, L. Gatti, S. Falasconi, and M. Stefanelli. A framework for building cooperative software agents in medical applications. Artificial Intelligence in Medicine, 16(3):223-249, 1999.

14. A. Lin. Integrating policy-driven role-based access control with common data secutiry architecture. Technical Report HPL-1999-59, HP Laboratories Bristol, 1999.

15. Ley Orgánica 15/1999 de protección de datos de carácter personal. Boletín Oficial del Estado 292, 14 de diciembre 1999.

16. A. López-Navidad, J. Kulisevsky, and F. Caballero, editors. El donante de órganos y tejidos: Evaluación $y$ manejo. Springer-Verlag Ibérica, $1^{\text {st }}$ edition, 1997.

17. R. Matesanz. Meeting the organ shortage: Current status and strategies for improvement of organ donation. Newsletter Transplant, 4(1):5-17, 1999.

18. Internet Medical Terminology Ressources. http://ai.bpa.arizona.edu.

19. S. Miksch. Plan management in medical domain. Ai. Communications, 4:209-235, 1999

20. The National Eye Institute . http://www.nei.nih.gov.

21. D.C. North. Institutions, Instutional Change and Econonomic Performance. Political Economy of Institutions and Decisions. Cambridge Univ. Press, $1^{\text {st }}$ edition, 1990.

22. D.M O'Day and J.M. Khoury. Donor tissue selection, pages 869-875. In Brightbill [4], 1999.

23. D. E. Oliver, Y. Shahar, M. A. Musen, and E. H. Shortliffe. Representation of change in controlled medical terminologies. Artificial Intelligence in Medicine, 15(1):53-76, 1999.

24. National Research Council. Committee on Maintaining Privacy and Security in Health Care Applications of the National Information Infrastructure, editors. For the Record: Protecting Electronic Health Information. National Academy Press, $1^{\text {st }}$ edition, 1997.

25. Organización Nacional de Transplantes. http://www.msc.es/ont.
26. E. Pattison-Gordon, J. J. Cimino, G. Hripsak, S. W. Tu, J. H. Gennari, N. L. Jain, and R. A. Greenes. Requirements of a sharable guideline representation for computer applications. Technical Report SMI-960628, Stanford Medical Institute, 1996.

27. M. J. Prietula, K. M. Carley, and L. Gasser, editors. Simulating Organizations. Computational Models of Institutions and Groups. AAAI Press/MIT Press, $1^{\text {st }}$ edition, 1998.

28. Real Decreto 994/1999 por el que se aprueba el reglamento de medidas de seguridad de los ficheros automatizados que contengan datos de carácter personal. Boletín Oficial del Estado 49, 26 de Febrero 2000 .

29. A. Rector, S. Bechhofer, C.A. Goble, I. Horrocks, W.A. Nolan, and W.D. Solomon. The GRAIL concept modelling language for medical terminology. Artificial Intelligence in Medicine, 9:139-171, 1997.

30. V. Shankararaman, V. Ambrosiaudou, T. Panchal, and B. Robinson. Agents in health care. In V. Shankararaman, editor, Workshop on Autonomus Agents in Health Care, pages 1-11. ACM-AAAI, ACM, 2000 .

\section{Acknowledgements}

We acknowledge the implementation efforts of Jorge Castillo (Carrel1), Jordi Angli (Carrel2) and Marc Font (Carrel3). We would like to thank Dr. Julian Padget for his help in the inception of this article. At last but not the least we like to acknowledge the three anonymous reviewers that have provided us with very valuable comments and constructive suggestions to enhance this paper.

This paper was supported in part by the EU research project Advanced Training System for Emergency Management (A-TEAM) IST-199910176 .

Ulises Cortés is a researcher of the Technical University of Catalonia (UPC) since 1982 (tenure 1988) working on several areas of Artificial Intelligence in the Software Department. He published more than 30 papers in scientific journals in the last 10 years. $\mathrm{He}$ is the coordinator of the Artificial Intelligence $\mathrm{Ph}$. D. Program of the UPC since 1991. In the last 10 years he and his group have been applying their work on AI to Environmental Decision Support Systems 
in special to Wastewater Treatment Plants. He has been awarded with the CLUSTER chair for 1998-1999 at the École Polytecnique Fédérale de Lausanne. See http://www.lsi.upc.es/ ia

Javier Váquez-Salceda is a $\mathrm{Ph}$. $\mathrm{D}$. student at the Technical University of Catalonia (UPC) who belongs to the Knowledge Engineering and Machine Learning Group (KEMLg) since 1999. His research is focused on the use of software agents technology and several other AI techniques to assist humans in complex domains such as Environmental Emergency Management, Collaborative Work or Resource Allocation. See http://www.lsi.upc.es/ jvazquez

Antonio López-Navidad School of Medicine, University of Málaga 1975-1981. Specialist in Internal
Medicine, Hospital de la Santa Creu i Sant Pau 19831987, Barcelona. Physician staff of the Department of Emergency and Critical Medicine, Hospital de la Santa Creu i Sant Pau 1988-1994. Director Program of Organ and Tissue Procurement for Transplantation, Hospital de la Santa Creu i Sant Pau since 1990. Director of the Tissue Bank, Hospital de la Santa Creu i Sant Pau since 1999. Associated Professor at the School of Medicine, Universidad Autńoma de Barcelona since 1996.

Francisco Caballero School of Medicine, University of Barcelona 1978-1984. Specialist in Internal Medicine, Hospital de la Santa Creu i Sant Pau 1987-1991, Barcelona. Physician of the Department of Emergency and Critical Medicine, Hospital de la Santa Creu i Sant Pau 1992-1994. Physician staff of the Department of Organ and Tissue Procurement for Transplantation, Hospital de la Santa Creu i Sant Pau since 1995. Director-attached of Tissue Bank, Hospital de la Santa Creu i Sant Pau since 1999. 